\title{
TILED ORDERS OF FINITE GLOBAL DIMENSION
}

\author{
HISAAKI FUJITA \\ Dedicated to Professor Hiroyuki Tachikawa on his 60 th birthday
}

\begin{abstract}
We define a projective link between maximal ideals, with respect to which an idealizer preserves being of finite global dimension. Let $D$ be a local Dedekind domain with the quotient ring $K$. We show that for $2 \leq n \leq 5$, every tiled $D$-order of finite global dimension in $(K)_{n}$ is obtained by iterating idealizers w.r.t. projective links from a hereditary order. For $n \geq 6$, we give a tiled $D$-order in $(K)_{n}$ without this property, which is also a counterexample to Tarsy's conjecture, saying that the maximum finite global dimension of such an order is $n-1$.
\end{abstract}

\section{INTRODUCTION}

Let $D$ be a local Dedekind domain with a unique maximal ideal $\pi D$ and the quotient ring $K$. If a $D$-order $\Lambda$ in the full $n \times n$ matrix ring $(K)_{n}$ contains $n$ orthogonal idempotents then $\Lambda$ is isomorphic to a subring $\left(\pi^{\lambda_{i j}} D\right)$ of $(D)_{n}$ where $\lambda_{i j} \geq 0, \lambda_{i i}=0$ and $\lambda_{i j}+\lambda_{j k} \geq \lambda_{i k}$ for any $1 \leq i, j, k \leq n$, and so $\Lambda$ is called a tiled $D$-order in $(K)_{n}$ (cf. [5]).

In [2] we have introduced an idealizer with respect to a link between maximal ideals in an arbitrary ring and shown that every tiled $D$-order is obtained by iterating the idealizers w.r.t. links of maximal ideals from a hereditary order. In this paper we define a projective link between maximal ideals, with respect to which an idealizer preserves being of finite global dimension (Theorem 1.1) and show that for $2 \leq n \leq 5$, every tiled $D$-order of finite global dimension in $(K)_{n}$ is obtained by iterating the idealizers w.r.t. projective links from a herediatry order (Theorem 2.1). This fact is deeply connected with the following conjecture posed by V. A. Jategaonkar [5]:

(J) If $\Lambda$ is a basic tiled $D$-order of finite global dimension then there is a vertex $i$ in the quiver of $\Lambda$ such that $\left|i^{+}\right|=1$ or $\left|i^{-}\right|=1$.

As noted in [5], if $(\mathbf{J})$ were true it would be also true that

(T) the maximum finite global dimension of a $D$-order in $(K)_{n}$ is $n-1$, holds for the class of tiled $D$-orders. This was conjectured by Tarsy [8]. For some classes of tiled $D$-orders, $(\mathrm{T})$ is settled by Jategaonkar [4, 5] and Kirkman

Received by the editors July 5, 1988 and, in revised form, November 29, 1988.

1980 Mathematics Subject Classification (1985 Revision). Primary 16A18; Secondary 16A60.

The main result in this paper was announced by the author at the Oberwolfach meeting on Orders and their applications from May 29 to June 4, 1988. 
and Kuzmanovich [6], and in [6] a counterexample to (J) (but not to (T)) is obtained. We shall give a counterexample to $(\mathrm{T})$ in the class of tiled $D$-orders in $(K)_{n}$ where $n \geq 6$ (Example 2.5). When $2 \leq n \leq 5$, (J) holds and using Theorem 2.1, a list of the representatives of isomorphism classes of tiled $D$ orders of finite global dimension in $(K)_{n}$ is obtained (Appendix).

We shall note that for the class of tiled $D$-orders of global dimension two, (J) holds. This gives some connection between these $D$-orders and maximal $D$-orders (Proposition 2.8).

Throughout this paper every ring is associative with an identity element and every module is unital.

The right (left) global dimension of a ring $R$ is denoted by r.gl.dim $R$ (1.gl.dim $R$ ). Let $R$ be an order in a simple artinian ring $Q$. Let $I, J$ be subsets of $Q$. Then we put $(I: J)_{r}=\{q \in Q \mid J q \subset I\},(I: J)_{l}=\{q \in Q \mid q J \subset I\}$, $O_{r}(I)=(I: I)_{r}$ and $O_{l}(I)=(I: I)_{l}$.

\section{AN IDEALIZER W.R.T. A LINK}

Let $S$ be a ring and $A$ a right ideal of $S$. Then the idealizer of $A$ in $S$ is the subring $\{s \in S \mid s A \subset A\}$ and denoted by $\mathbf{I}_{S}(A) . A$ is said to be generative if $S A=S$ holds. A subring $R$ of $\mathbf{I}_{S}(A)$ containing $A$ is a tame subidealizer if $A$ is a generative right ideal of $S$ and if $R / A$ is a semisimple artinian ring (cf. [3]). We also define similar notions for a left ideal. Let $M_{a}, M_{b}$ be maximal ideals of $S$. Then there is a link from $M_{a}$ to $M_{b}$, denoted by $M_{a} \rightsquigarrow M_{b}$, if $M_{a} \cap M_{b} \supsetneqq M_{b} M_{a}$ and if $S / M_{a}$ and $S / M_{b}$ are artinian. When $M_{a} \rightsquigarrow M_{b}$ and $M_{a} \neq M_{b}$, there exists a right ideal $A$ of $S$ such that

$$
M_{b} / M_{b} M_{a}=A / M_{b} M_{a} \oplus\left(M_{a} \cap M_{b}\right) / M_{b} M_{a} .
$$

The idealizer of $A$ in $S$ is called an idealizer with respect to a link $M_{a} \rightsquigarrow M_{b}$. It should be noted that if $\Lambda \supset \Gamma$ are successive tiled $D$-orders then $\Gamma$ is obiained as an idealizer w.r.t. a link in $\Lambda$ (cf. [2, §4]).

Utilizing tame subidealizers, we can obtain bounds of global dimension of an idealizer w.r.t. a certain link.

Theorem 1.1. Let $S$ be a ring and $M_{a}, M_{b}$ maximal ideals of $S$ such that $M_{a} \leadsto M_{b}$ and $M_{b} \nrightarrow M_{a}$, and let $R$ be an idealizer w.r.t. $M_{a} \rightsquigarrow M_{b}$. Suppose that there exists a ring $T$ such that $S$ is contained in $T$ as a subring and that $M_{b}$ is a generative right ideal of $T$ (or $M_{a}$ is a generative left ideal of $T$ ). Then

$$
\text { r.gl.dim } S-1 \leq \text { r.gl.dim } R \leq \text { r.gl.dim } S+1
$$

and

$$
\text { 1.gl.dim } S-1 \leq \text { 1.gl.dim } R \leq 1 . \text { gl.dim } S+1 \text {. }
$$

Proof. It follows from [2, Lemma 1.1] that there are a right ideal $A$ and a left ideal $B$ of $S$ such that $M_{b} \supset A \supset M_{b} M_{a}, M_{a} \supset B \supset M_{b} M_{a}$,

$$
M_{b} / M_{b} M_{a}=A / M_{b} M_{a} \oplus\left(M_{a} \cap M_{b}\right) / M_{b} M_{a},
$$




$$
M_{a} / M_{b} M_{a}=B / M_{b} M_{a} \oplus\left(M_{a} \cap M_{b}\right) / M_{b} M_{a}
$$

and that $R=\mathbf{I}_{S}(A)=\mathbf{I}_{S}(B)$. Note that $M_{b} M_{a}=A \cap B$. Hence by [2, Corollary 1.4], $M_{b} / M_{b} M_{a}$ is semisimple as a left $R$-module. Since $B\left(A / M_{b} M_{a}\right)=0$ and $A\left(\left(M_{a} \cap M_{b}\right) / M_{b} M_{a}\right)=0, A / M_{b} M_{a}$ is a homogeneous component, so fully invariant in $M_{b} / M_{b} M_{a}$ as left $R$-modules. Since $M_{b} \not 4 M_{a}, M_{a} M_{b}=$ $M_{a} \cap M_{b} \supset M_{b} M_{a}$. Suppose that $M_{b}$ is a generative right ideal of $T$. Then note that $M_{b}^{2}=M_{b} T M_{b}=M_{b} T=M_{b}$. Hence

$$
M_{b} M_{a} \supset M_{b} M_{a} M_{b} T \supset M_{b} M_{b} M_{a} T=M_{b} M_{a} T \supset M_{b} M_{a},
$$

so that $M_{b} M_{a}=M_{b} M_{a} T$. Therefore for any $t \in T, f_{t}: x+M_{b} M_{a} \mapsto x t+M_{b} M_{a}$ is a well-defined mapping in $\operatorname{End}_{R}\left(M_{b} / M_{b} M_{a}\right)$. Hence

$$
\left(A t+M_{b} M_{a}\right) / M_{b} M_{a}=f_{t}\left(A / M_{b} M_{a}\right) \subset A / M_{b} M_{a}
$$

and thus $A$ is a right ideal of $T$. Moreover by [2, Lemma 1.1(b)], TA=TSA= $T M_{b}=T$. Therefore $A$ is a generative right ideal of $T$. In a similar way, we can show that if $M_{a}$ is a generative left ideal of $T$ then so is $B$. Consequently $S$ and $R$ are tame subidealizers in $T$. It is now easy to complete the proof by [3, Theorems 2.2 and 2.10].

When $S$ is a noetherian prime ring, the theorem is rephrased as follows.

Corollary 1.2. Let $S$ be a noetherian prime ring and $M_{a}, M_{b}$ maximal ideals of $S$ with $M_{a} \rightsquigarrow M_{b}$ and $M_{b} \leftrightarrow M_{a}$, and let $R$ be an idealizer w.r.t. $M_{a} \rightsquigarrow M_{b}$. If $M_{b}$ is idempotent and left $S$-projective (or $M_{a}$ is idempotent and right $S$ projective), then

$$
\text { gl.dim } S-1 \leq \text { gl.dim } R \leq \operatorname{gl} \cdot \operatorname{dim} S+1 .
$$

Proof. Recall that a noetherian prime ring is an order in a simple artinian ring. Put $T=O_{r}\left(M_{b}\right)$. Suppose that $M_{b}$ is idempotent and left $S$-projective. Then $T=\left(S: M_{b}\right)_{r}$ and $\left(S: M_{b}\right)_{r} M_{b}=T$, so that $M_{b}$ is a generative right ideal of $T$ (and vice versa (cf. [3, Proposition 1.1]). Thus $T$ satisfies the hypotheses of the theorem. This completes the proof.

Definition. We call a link between maximal ideals projective if it satisfies the hypotheses of the theorem.

Remarks. (1) In [8], Tarsy conjectured that if $\Lambda \supset \Gamma$ are successive $D$-orders of finite global dimension then their global dimensions differ by at most one. A counterexample to this conjecture is given in [4]. However, if $\Lambda$ and $\Gamma$ are basic tiled $D$-orders and if the associated link is projective then this conjecture follows from the theorem.

(2) The hypothesis ' $M_{b} \not \hookrightarrow M_{a}$ ' is necessary (cf. [2, Proposition 5.6]).

(3) In the theorem, gl.dim $S-1$ and gl.dim $S+1$ are the best bounds of gl.dim $R$. 
Example 1.3. Let $\Lambda$ be the basic tiled $D$-order

$$
\left(\begin{array}{ccccc}
D & \pi D & \pi D & \pi D & \pi D \\
D & D & \pi D & \pi D & \pi D \\
D & D & D & \pi D & \pi D \\
\pi D & \pi D & \pi D & D & \pi D \\
D & D & \pi D & D & D
\end{array}\right)
$$

Then gl.dim $\Lambda=3$ and the link graph of $\Lambda$ is given by

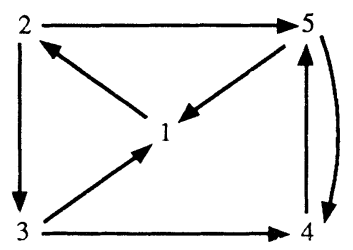

Let $M_{i}$ be the set of all matrices in $\Lambda$ whose $(i, i)$-entries are in $\pi D \quad(i=$ $1, \ldots, 5)$. Then $M_{1}, \ldots, M_{5}$ are the maximal ideals of $\Lambda$. Note that $M_{1}$ and $M_{4}$ are left $\Lambda$-projective. Let $\alpha_{1}, \alpha_{2}$, and $\alpha_{3}$ be the links $M_{3} \rightsquigarrow M_{4}, M_{3} \rightsquigarrow$ $M_{1}$, and $M_{5} \rightsquigarrow M_{1}$, respectively, and let $\Gamma_{i}$ be the idealizer w.r.t. $\alpha_{i}(i=$ $1,2,3)$. Then gl.dim $\Gamma_{1}=2$, gl.dim $\Gamma_{2}=3$, and gl.dim $\Gamma_{3}=4$.

\section{TILED ORDERS OF FINITE GLOBAL DIMENSION}

Let $D$ be a local Dedekind domain with a unique maximal ideal $\pi D$ and the quotient ring $K$. The main object of this section is to prove

Theorem 2.1. Suppose that $2 \leq n \leq 5$. Let $\Lambda$ be a basic tiled $D$-order in $(K)_{n}$ and gl.dim $\Lambda<\infty$. Then there exists a chain of basic tiled D-orders $\Lambda=\Lambda_{0} \subset \Lambda_{1} \subset \cdots \subset \Lambda_{t}$ such that $\Lambda_{t}$ is hereditary and that $\Lambda_{i-1}$ is the idealizer w.r.t. a projective link in $\Lambda_{i}$ where $i=1, \ldots, t$. If $n \geq 6$ then there exists a tiled D-order of finite global dimension in $(K)_{n}$ which does not have the above property.

We now recall some definitoins and notation of tiled $D$-orders. Let $n \geq$ 2 and let $\Lambda=\left(\pi^{\lambda_{i j}} D\right)$ be a basic tilded $D$-order in $(K)_{n}$, where $\lambda_{i j}$ 's are nonnegative integers, $\lambda_{i i}=0, \lambda_{i j}+\lambda_{j k} \geq \lambda_{i k}$, and $\lambda_{i j}+\lambda_{j i}>0$ (if $i \neq j$ ) for any $1 \leq i, j, k \leq n$. Put $m_{k i j}=1$ (if $i=j=k$ ) and $\lambda_{i j}$ (otherwise) for any $1 \leq i, j, k \leq n$ and $M_{k}=\left(\pi^{m_{k i j}} D\right)$ for any $1 \leq k \leq n$. Then $M_{1}, \ldots, M_{n}$ are the maximal ideals of $\Lambda$ and $J=M_{1} \cap \cdots \cap M_{n}$ is the Jacobson radical of $\Lambda$. Let $e_{i}$ be the matrix in $(K)_{n}$ whose $(i, i)$-entry is 1 and the others are 0 . $\mathscr{Q}(\Lambda)=\left(\mathscr{Q}(\Lambda)_{0}, \mathscr{Q}(\Lambda)_{1}, v\right)$ is the valued quiver of $\Lambda$ provided that $\mathscr{Q}(\Lambda)_{0}=\{1, \ldots, n\}$ is the set of vertices, $\mathscr{Q}(\Lambda)_{1}$ is the set of arrows defined by $i \rightarrow j \in \mathscr{Q}(\Lambda)_{1}$ if $e_{j}\left(J / J^{2}\right) e_{i} \neq 0$ and that $v$ is the mapping from $\mathscr{Q}(\Lambda)_{1}$ to nonnegative integers such that for any $\alpha: i \rightarrow j \in \mathscr{Q}(\Lambda)_{1}, v(\alpha)=\lambda_{j i}($ if $i \neq j)$ and 1 (if $i=j$ ). Let

$$
p: x_{0} \stackrel{\alpha_{1}}{\longrightarrow} x_{1} \stackrel{\alpha_{2}}{\longrightarrow} \cdots \stackrel{\alpha_{m}}{\longrightarrow} x_{m}
$$


be a path in $\mathscr{Q}(\Lambda)$. Then we put $v(p)=\sum_{i=1}^{m} v\left(\alpha_{i}\right)$ and we call $p$ a $v(p)$-path. $\mathscr{P}(\Lambda)$ denotes the set of all $\lambda_{i j}$-paths from $j$ to $i$ in $\mathscr{Q}(\Lambda)$ where $i, j \in \mathscr{Q}(\Lambda)_{0}$ and $i \neq j$. An order is associated with $\mathscr{P}(\Lambda)$, i.e., for $p_{1}, p_{2} \in \mathscr{P}(\Lambda), p_{1} \leq p_{2}$ if $p_{1}$ is a subpath of $p_{2}$. For $i \in \mathscr{Q}(\Lambda)_{0}, i^{+}$(resp. $i^{-}$) denotes the set $\left\{x \in \mathscr{Q}(\Lambda)_{0} \mid i \rightarrow x \in \mathscr{Q}(\Lambda)_{1}\right\}$ (resp. $\left.\left\{y \in \mathscr{Q}(\Lambda)_{0} \mid y \rightarrow i \in \mathscr{Q}(\Lambda)_{1}\right\}\right)$. Note that $M_{i} \rightsquigarrow M_{j}$ if and only if $i \rightarrow j \in \mathscr{Q}(\Lambda)_{1}$. (See [9] and [2, §4].)

Let $I=\left(\pi^{a_{i j}} D\right)$ and $H=\left(\pi^{b_{i j}} D\right)$ be ideals of $\Lambda$, and put

$$
x_{i j}=\operatorname{Max}\left\{b_{k j}-a_{k i} \mid 1 \leq k \leq n\right\}
$$

and

$$
y_{i j}=\operatorname{Max}\left\{b_{i k}-a_{j k} \mid 1 \leq k \leq n\right\}
$$

for any $1 \leq i, j \leq n$. Then it holds that $(H: I)_{r}=\left\{q \in(K)_{n} \mid I q \subset H\right\}=\left(\pi^{x_{i j}} D\right)$ and $(H: I)_{l}=\left\{q \in(K)_{n} \mid q I \subset H\right\}=\left(\pi^{y_{i j}} D\right)$.

Lemma 2.2. Let $M_{b}$ be a maximal ideal of $\Lambda$ and put $\Gamma=O_{r}\left(M_{b}\right)$. Then $\Lambda=\mathbf{I}_{\Gamma}\left(M_{b}\right)$.

Proof. Put $\Gamma=\left(\pi^{\gamma_{i j}} D\right)$ and $O_{l}\left(M_{b}\right)=\left(\pi^{y_{i j}} D\right)$. Since $\Lambda \subset O_{r}\left(M_{b}\right) \cap O_{l}\left(M_{b}\right)$, $\gamma_{i j} \leq \lambda_{i j}$ and $y_{i j} \leq \lambda_{i j}$ for any $1 \leq i, j \leq n$. It is easily checked that $\gamma_{i j} \geq \lambda_{i j}$ (if $i \neq b$ ) and $y_{i j} \geq \lambda_{i j}$ (if $j \neq b$ ). This completes the proof.

Lemma 2.3. Let $M_{b}$ be a maximal ideal of $\Lambda$ and projective as a left $\Lambda$-module. Put $\Gamma=O_{r}\left(M_{b}\right)=\left(\pi^{\gamma_{i j}} D\right)$. Then there exists $l \in \mathscr{Q}(\Lambda)_{0}$ such that $b^{+}=\{l\}$ and $l \neq b$. Moreover,

(1) for any $1 \leq i \leq n, m_{b i b}=m_{b i l}+m_{b l b}$, in particular $\lambda_{b l}+\lambda_{l b}=1$.

(2) $\gamma_{i j}=\lambda_{l j}-\lambda_{l b}($ if $i=b)$ and $\lambda_{i j}$ (otherwise).

Proof. Since $M_{b}$ is left $\Lambda$-projective, $b^{+}=\{l\}$ for some $l \in \mathscr{Q}(\Lambda)_{0}$ with $l \neq b$.

(1) Let $i \neq b$. Then any $\lambda_{i b}$-path from $b$ to $i$ contains the arrow $b \rightarrow l$. Hence $\lambda_{i b}=\lambda_{i l}+\lambda_{l b}$ and so $m_{b i b}=m_{b i l}+m_{b l b}$. Let $i=b$. Then for any $k \neq b, l, \lambda_{b k}+\lambda_{k b}=\lambda_{b k}+\lambda_{k l}+\lambda_{l b} \geq \lambda_{b l}+\lambda_{l b}$. Since $b \rightarrow b \notin \mathscr{Q}(\Lambda)_{1}$,

$$
1=m_{b b b}=\operatorname{Min}\left\{m_{b b k}+m_{b k b} \mid 1 \leq k \leq n\right\}=\lambda_{b l}+\lambda_{l b} \text {. }
$$

This completes the proof of (1).

(2) This follows from (1) and the proof of Lemma 2.2.

A right ideal of a ring is semimaximal if it is an intersection of finitely many maximal right ideals.

Lemma 2.4. Let $M_{b}$ be left $\Lambda$-projective with $b^{+}=\{l\}$ and $\Gamma=O_{r}\left(M_{b}\right)$. Then the following statements are equivalent.

(1) $l^{-}=\{b\}$.

(2) $\Gamma$ is left $\Lambda$-projective.

(3) $M_{b}$ is a (semi)maximal right ideal of $\Gamma$.

Proof. $(1) \Rightarrow(2)$. Let $j \neq b, l$. Then by the hypothesis, $\lambda_{l j}=\lambda_{l b}+\lambda_{b j}$. Hence $\lambda_{l j}-\lambda_{l b}=\lambda_{b j}$. Hence by Lemma 2.3(2), $\Gamma e_{j}=\Lambda e_{j}$. Moreover, by Lemma 2.3, $\Gamma e_{b}=\Lambda e_{b}$ and $\Gamma e_{l} \cong \Lambda e_{b}$, so that $\Gamma$ is left $\Lambda$-projective. 
(2) $\Rightarrow(1)$. Since ${ }_{\Lambda} \Gamma$ is finitely generated and projective, $\Gamma / M_{b} \cong \Lambda / M_{b} \otimes_{\Lambda} \Gamma$ is simple as a right $\Gamma$-module. By Lemma $2.2, \Lambda=\mathrm{I}_{\Gamma}\left(M_{b}\right)$. Thus it follows from [7, Theorem 1.3] that $0 \rightarrow \Lambda / M_{b} \rightarrow \Gamma / M_{b} \rightarrow \Gamma / \Lambda \rightarrow 0$ is a nonsplit exact sequence of right $\Lambda$-modules and $(\Gamma / \Lambda)_{\Lambda}$ is simple. Since $b^{+}=\{l\}$, $M_{l}=\operatorname{ann}_{\Lambda}(\Gamma / \Lambda)=(\Lambda: \Gamma)_{r}$. Since ${ }_{\Lambda} \Gamma$ is projective, $M_{l} \Gamma=O_{r}(\Gamma)=\Gamma$. Hence $M_{l}$ is right $\Lambda$-projective, so that $l^{-}=\{b\}$.

$(2) \Leftrightarrow(3)$. This follows from [3, Proposition 1.3] and Lemma 2.2.

Let $n_{0}$ be the maximum number among $n$ 's satisfying the following condition:

(J) If $\Lambda$ is a basic tiled $D$-order in $(K)_{n}$ and gl.dim $\Lambda<\infty$ then there exists $i \in \mathscr{Q}(\Lambda)_{0}$ such that $\left|i^{+}\right|=1$ or $\left|i^{-}\right|=1$.

It follows from [ 5 , Theorem $4.2 ; 4$, Lemma 5 ] that $n_{0} \geq 4$. The following example shows that $n_{0} \leq 5$, which is also a counterexample to Tarsy's conjecture.

Example 2.5. Let $\Lambda$ be the basic tiled $D$-order whose valued quiver is given by

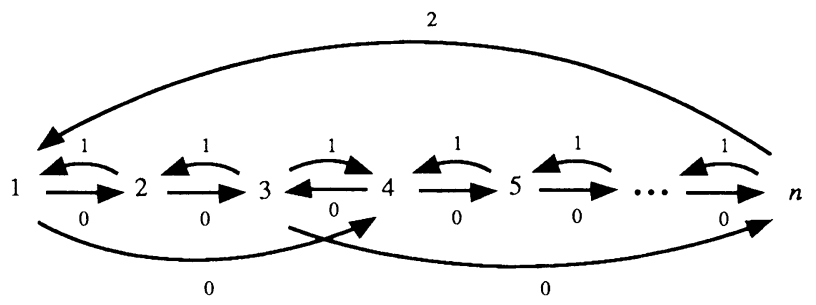

here $n \geq 6$. Then gl.dim $\Lambda=n$ and $(\mathrm{J})$ does not hold.

Remarks. (1) This example is something like Tarsy's [8, Theorem 11].

(2) It is shown in [5] that for a fixed $n$, there are only finitely many isomorphism classes of tiled $D$-orders of finite global dimension in $(K)_{n}$. Hence there is the maximum finite global dimension of a tiled $D$-order in $(K)_{n}$. Although, at present, $n$ is the maximum among known examples, we do not have enough reason to conjecture that $n$ is the maximum.

By [5, Theorem 2.3], we can determine whether $n_{0}$ is 4 or 5 at least in principle. Making use of the idealizers w.r.t. links [2, Proposition 4.6], we can shorten the computation and it is verified that when $n=5,(\mathrm{~J})$ holds. Thus we obtain

Proposition 2.6. The maximum number satisfying ( $\mathbf{J})$ is 5 .

Lemma 2.7. Let $\Lambda$ be a basic tiled $D$-order in $(K)_{n}$ and $M_{b}$ a maximal ideal of $\Lambda$. Then the following statements are equivalent.

(1) $M_{b}$ is left $\Lambda$-reflexive.

(2) $O_{r}\left(M_{b}\right) \supsetneqq \Lambda$.

(3) There exists $a \in \mathscr{Q}(\Lambda)_{0}$ such that $a \neq b$ and any $\lambda_{b a}$-path from $a$ to $b$ is maximal in $\mathscr{P}(\Lambda)$. 
Proof. Since $M_{b}$ is a maximal ideal, $M_{b}$ is left $\Lambda$-reflexive if and only if $\left(\Lambda:\left(\Lambda: M_{b}\right)_{r}\right)_{l} \varsubsetneqq \Lambda$ if and only if $\left(\Lambda: M_{b}\right)_{r} \supsetneqq \Lambda$. As in the proof of Lemma 2.2, it is shown that $O_{r}\left(M_{b}\right)=\left(\Lambda: M_{b}\right)_{r}$. Hence (1) is equivalent to (2).

Put $O_{r}\left(M_{b}\right)=\left(\pi^{\gamma_{i j}} D\right)$. If $i \neq b$ then $\gamma_{i j}=\lambda_{i j}$ holds. Hence $O_{r}\left(M_{b}\right) \supsetneqq \Lambda$ if and only if there is $a \in \mathscr{Q}(\Lambda)_{0}$ such that $a \neq b$ and $\lambda_{b a}>\operatorname{Max}\left\{m_{b k a}-m_{b k b} \mid 1 \leq\right.$ $k \leq n\}$ if and only if there is $a \in \mathscr{Q}(\Lambda)_{0}$ such that $a \neq b$ and $\lambda_{b a}+\lambda_{k b}>\lambda_{k a}$ for any $k \neq a, b$. Retaking $a$ if necessary, this is equivalent to (3). This completes the proof.

Although the next proposition deviates from the proof of the theorem, we obtain it as an application of Lemma 2.7.

Proposition 2.8. Let $\Lambda$ be a basic tiled D-order in $(K)_{n}$ and $\operatorname{gl}$.dim $\Lambda=2$. Then there exist $a, b \in \mathscr{Q}(\Lambda)_{0}$ such that $\left|a^{-}\right|=1$ and $\left|b^{+}\right|=1$. Moreover, there exists a chain of tiled D-orders $\Lambda=\Gamma_{0} \subset \Gamma_{1} \subset \cdots \subset \Gamma_{m}$ such that $\Gamma_{m}$ is a maximal order, $\Gamma_{i-1}$ is obtained as an idealizer in $\Gamma_{i}(1 \leq i \leq m)$ and that for some $1 \leq l \leq m$, gl.dim $\Gamma_{i}=1$ (if $l \leq i \leq m$ ) and 2 (if $\left.0 \leq i<l\right)$.

Proof. Since $\mathscr{P}(\Lambda)$ is a finite set, there exist $a, b \in \mathscr{Q}(\Lambda)_{0}$ such that any $\lambda_{b a^{-}}$ path from $a$ to $b$ in $\mathscr{Q}(\Lambda)$ is maximal in $\mathscr{P}(\Lambda)$. Hence by Lemma $2.7, M_{a}$ (resp. $M_{b}$ ) is right (resp. left) $\Lambda$-reflexive. Since gl.dim $\Lambda=2$, it follows from [1, Proposition 5.2] that $M_{a}$ (resp. $M_{b}$ ) is right (resp. left) $\Lambda$-projective, i.e., $\left|a^{-}\right|=1$ and $\left|b^{+}\right|=1$. Put $\Gamma_{1}=O_{r}\left(M_{b}\right)$. Then $\Gamma_{1}$ is finitely generated and projective as a right $\Lambda$-module. Hence gl.dim $\Gamma_{1} \leq$ gl.dim $\Lambda$. By Lemma 2.2, $\Lambda=\mathbf{I}_{\Gamma_{1}}\left(M_{b}\right)$. Repeating a similar argument, we obtain a desired chain.

We are now in a position to prove the theorem.

Proof of Theorem 2.1. Let $\Lambda$ be a basic tiled $D$-order in $(K)_{n}$ with $a \rightarrow b \in$ $\mathscr{Q}(\Lambda)_{1}$ and $a \neq b$, and let $\Gamma$ be the idealizer w.r.t. $a \rightarrow b$ in $\Lambda$. Then it follows from [2, Theorem 4.9] that the new arrows in $\mathscr{Q}(\Gamma)_{1}$ are in the form of $a \rightarrow y$ or $x \rightarrow b$ where $x \in a^{-}$and $y \in b^{+}$. Hence if $a \rightarrow b$ is a projective link then $\left|b^{+}\right|=1$ or $\left|a^{-}\right|=1$ in $\mathscr{Q}(\Gamma)$. Therefore any basic tiled $D$-order obtained by iterating the idealizers w.r.t. projective links from a hereditary order satisfies $(\mathrm{J})$. Hence for $n \geq 6$, the tiled $D$-order of Example 2.5 is not obtained in such a way.

Suppose that $2 \leq n \leq 5$. Let $\Lambda$ be a basic tiled $D$-order with gl.dim $\Lambda<\infty$. It follows from Proposition 2.6 that there exists $b \in \mathscr{Q}(\Lambda)_{0}$ such that $\left|b^{-}\right|=1$ or $\left|b^{+}\right|=1$. Let $b^{+}=\{l\}$. Put

$$
u=\left(\begin{array}{ccc}
\pi^{\lambda_{l 1}} & & 0 \\
& \ddots & \\
0 & & \pi^{\lambda_{l n}}
\end{array}\right)
$$

and $\Lambda^{\prime}=u \Lambda u^{-1}=\left(\pi^{\lambda_{i j}^{\prime}} D\right)$. Then $\lambda_{l j}^{\prime}=0$ for any $1 \leq j \leq n$ and $\lambda_{i j}^{\prime} \geq 0$ for any $1 \leq i, j \leq n$ (cf. [5, Lemma 11]). Note that $\lambda_{i j}^{\prime}+\lambda_{j i}^{\prime}=\lambda_{i j}+\lambda_{j i}$. Hence 
$\lambda_{b l}^{\prime}=\lambda_{b l}^{\prime}+\lambda_{l b}^{\prime}=\lambda_{b l}+\lambda_{l b}=1$ by Lemma 2.3(1). Hence for any $1 \leq j \leq n$, $0=\lambda_{l j}^{\prime} \leq \lambda_{l b}^{\prime}+\lambda_{b j}^{\prime}=\lambda_{b j}^{\prime} \leq \lambda_{b l}^{\prime}+\lambda_{l j}^{\prime}=1$, so that $\lambda_{b j}^{\prime}=0$ or 1 .

Suppose that $l^{-} \neq\{b\}$. Then for some $a$ with $a \neq b, l, \lambda_{b a}^{\prime}=1$. Since $b \rightarrow l \in \mathscr{Q}\left(\Lambda^{\prime}\right)_{1}, 0=\lambda_{l b}^{\prime}<\lambda_{l a}^{\prime}+\lambda_{a b}^{\prime}=\lambda_{a b}^{\prime}$. Hence $\lambda_{b a}^{\prime}+\lambda_{a b}^{\prime} \geq 2$. Since $M_{b}$ is left $\Lambda$-projective, it follows from Lemma 2.7 that retaking $a$ if necessary, any $\lambda_{b a}$-path $a$ to $b$ is maximal in $\mathscr{P}\left(\Lambda^{\prime}\right)$.

Put $\Lambda_{1}^{\prime}=\left(\pi^{\lambda_{1 i j}^{\prime}} D\right)$ where $\lambda_{1 i j}^{\prime}=\lambda_{i j}^{\prime}-1$ (if $\left.(i, j)=(b, a)\right)$ and $\lambda_{i j}^{\prime}$ (otherwise). Then it follows from [2, Lemmas 4.3 and 4.5] that $\Lambda_{1}^{\prime}$ is a basic tiled $D$-order and $\Lambda^{\prime}$ is the idealizer w.r.t. $a \rightarrow b$ in $\Lambda_{1}^{\prime}$. Put $\Gamma^{\prime}=O_{r}\left(M_{b}^{\prime}\right)$. Then $M_{1 b}^{\prime}=\Lambda_{1}^{\prime} M_{b}^{\prime}$ is a maximal ideal of $\Lambda_{1}^{\prime}$ by [2, Lemma 1.1], $\Gamma^{\prime}=O_{r}\left(M_{1 b}^{\prime}\right)$ and $\Gamma^{\prime} M_{1 b}^{\prime}=\Gamma^{\prime} M_{b}^{\prime}=\Gamma^{\prime}$. Therefore $M_{1 b}^{\prime}$ is left $\Lambda_{1}^{\prime}$-projective, so that $b^{+}=\{l\}$ in $\mathscr{Q}\left(\Lambda_{1}^{\prime}\right)$. If $b \rightarrow a \in \mathscr{Q}\left(\Lambda_{1}^{\prime}\right)_{1}$ then $a=l$, so $\lambda_{1 a b}^{\prime}=\lambda_{l b}^{\prime}=0$ and so $\lambda_{1 a b}^{\prime}+\lambda_{1 b a}^{\prime}=0$, which is a contradiction. Thus $a \rightarrow b$ is a projective link in $\Lambda_{1}^{\prime}$. It follows from Theroem 1.1 that gl.dim $\Lambda_{1}^{\prime}<\infty$.

After at most $n-2$ times repetition of the above procedure, we obtain a chain of basic tiled $D$-orders of finite global dimension $\Lambda^{\prime}=\Lambda_{0}^{\prime} \subset \Lambda_{1}^{\prime} \subset \cdots \subset \Lambda_{r}^{\prime}$ such that $\Lambda_{i-1}^{\prime}$ is the idealizer w.r.t. a projective link $a_{i} \rightarrow b$ in $\Lambda_{i}^{\prime} \quad(1 \leq i \leq r)$ and $\lambda_{r b j}^{\prime}=0$ for any $j \neq l$ where $\Lambda_{r}^{\prime}=\left(\pi^{\lambda_{r i j}^{\prime}} D\right)$. Hence $l^{-}=\{b\}$ in $\mathscr{Q}\left(\Lambda_{r}^{\prime}\right)$. When $\left|b^{-}\right|=1$, consider a similar argument as above. If $\Lambda_{r}^{\prime}$ is not hereditary, repeat the above argument. It is now easy to complete the proof by

Lemma 2.9. Suppose that $2 \leq n \leq 5$. Let $\Lambda$ be a basic tiled D-order in $(K)_{n}$ and gl.dim $\Lambda<\infty$. Then the following statements are equivalent.

(1) For any $1 \leq i, j \leq n$ with $i \neq j, i^{+}=\{j\}$ if and only if $j^{-}=\{i\}$.

(2) $\Lambda$ is a hereditary order.

Proof. It is clear that (2) implies (1). We prove $(1) \Rightarrow(2)$ by induction on $n$. If $n=2$ then gl.dim $\Lambda<\infty$ if and only if $\Lambda$ is hereditary, so that the assertion holds. Let $n \geq 3$. Then by Proposition 2.6 and the assumption, there exist $1 \leq b, l \leq n$ such that $b \neq l, b^{+}=\{l\}$ and $l^{-}=\{b\}$. Put $\Gamma=O_{r}\left(M_{b}\right)$ and $\Gamma^{\prime}=e \Gamma e$ where $e=\sum_{i \neq l} e_{i}$. Then $\Gamma^{\prime}$ satisfies (1) and by the induction hypothesis $\Gamma^{\prime}$ is hereditary. Since $\Gamma$ is Morita equivalent to $\Gamma^{\prime}$, $\Gamma$ is also hereditary. By Lemma $2.2, \Lambda=\mathbf{I}_{\Gamma}\left(M_{b}\right)$ and by Lemma $2.4, M_{b}$ is a semimaximal right ideal of $\Gamma$. Therefore it follows from [7, Theorem 4.3] that $\Lambda$ is a hereditary order. This completes the proof.

Corollary 2.10. Suppose that $2 \leq n \leq 5$. Then the set of basic tiled D-orders of finite global dimension in $(K)_{n}$ coincides with the set of basic tiled D-orders in $(K)_{n}$ obtained by finite iterations of the idealizers w.r.t. projective links from a hereditary order.

Proof. This follows from Theorems 1.1 and 2.1.

In connection with the corollary and [2, Proposition 4.6], it might be a natural question whether any basic tiled $D$-order of finite global dimension appears in a 
chain of successive tiled $D$-orders of finite global dimension from a hereditary order. The following example shows that this is not true.

Example 2.11. Let $\Lambda=\left(\pi^{\lambda_{i j}} D\right)$ be the basic tiled $D$-order

$$
\left(\begin{array}{cccccc}
D & D & D & D & D & D \\
\pi D & D & \pi D & \pi D & D & D \\
\pi D & \pi D & D & D & D & D \\
\pi^{2} D & \pi D & \pi^{2} D & D & \pi D & D \\
\pi^{2} D & \pi^{2} D & \pi D & \pi D & D & D \\
\pi^{2} D & \pi^{2} D & \pi^{2} D & \pi D & \pi D & D
\end{array}\right)
$$

Then gl.dim $\Lambda=5$. Let $\left(a_{1}, b_{1}\right)=(6,1),\left(a_{2}, b_{2}\right)=(4,2),\left(a_{3}, b_{3}\right)=(3,4)$, and $\left(a_{4}, b_{4}\right)=(2,5)$. For $1 \leq k \leq 4$ and $1 \leq i, j \leq 6$, put $\gamma_{k i j}=\lambda_{i j}-1$ (if $\left.(j, i)=\left(a_{k}, b_{k}\right)\right)$ and $\lambda_{i j}$ (otherwise) and put $\Gamma_{k}=\left(\pi^{\gamma_{k i j}} D\right)$. Then $\Gamma_{1}, \ldots, \Gamma_{4}$ are the successive tiled $D$-orders containing $\Lambda$ and gl.dim $\Gamma_{k}=\infty$ for all $k=1, \ldots, 4$.

\section{APPENDIX}

In this appendix we shall give a list of the representatives of isomorphism classes of tiled $D$-orders of finite global dimension in $(K)_{n}$ where $n=4,5$. This list is obtained by computation using Theorem 2.1. When $n \leq 3$, see [8].

Let $\Lambda=\left(\pi^{\lambda_{i j}} D\right)$ be a tiled $D$-order. Then we put $d=d(\Lambda)=\sum_{i, j} \lambda_{i j}$. In the following matrices $\Lambda, 2^{i}$ stands for $\pi^{i} D(0 \leq i \leq 4)$.

$$
d=13
$$

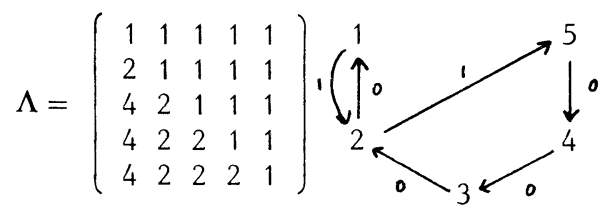

$\operatorname{gl} \cdot \operatorname{dim} \Lambda=2$

$d=13$

$$
\Lambda=\left(\begin{array}{lllll}
1 & 1 & 1 & 1 & 1 \\
2 & 1 & 1 & 1 & 1 \\
2 & 2 & 1 & 1 & 1 \\
4 & 2 & 2 & 1 & 2 \\
4 & 2 & 2 & 2 & 1
\end{array}\right)
$$

$\operatorname{gl} \operatorname{dim} \Lambda=2$

$$
d=13
$$

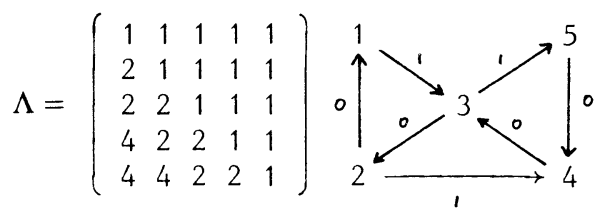

gl. $\operatorname{dim} \Lambda=2$

$$
d=14
$$

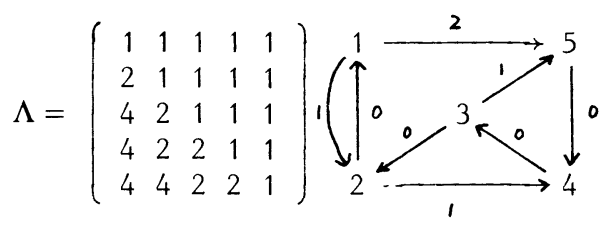

$\operatorname{gl} \cdot \operatorname{dim} \Lambda=3$ 
$d=14$

$d=14$

$\Lambda=\left(\begin{array}{lllll}1 & 1 & 1 & 1 & 1 \\ 2 & 1 & 2 & 1 & 1 \\ 4 & 2 & 1 & 1 & 1 \\ 4 & 2 & 2 & 1 & 1 \\ 4 & 2 & 2 & 2 & 1\end{array}\right),(\int_{2}^{1} \stackrel{0}{\longleftarrow} \overbrace{0}^{5} \coprod_{4}^{3}$

$\Lambda=\left(\begin{array}{lllll}1 & 1 & 1 & 1 & 1 \\ 2 & 1 & 1 & 1 & 1 \\ 4 & 2 & 1 & 2 & 1 \\ 4 & 2 & 2 & 1 & 1 \\ 4 & 2 & 2 & 2 & 1\end{array}\right)$

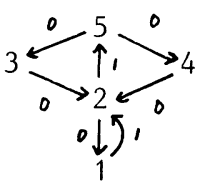

gl. $\operatorname{dim} \Lambda=3$

gl.dim $\Lambda=2$

$d=14$

$d=14$

$\Lambda=\left(\begin{array}{lllll}1 & 1 & 1 & 1 & 1 \\ 2 & 1 & 1 & 1 & 1 \\ 4 & 2 & 1 & 1 & 1 \\ 4 & 2 & 2 & 1 & 2 \\ 4 & 2 & 2 & 2 & 1\end{array}\right)$

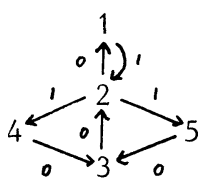

$\operatorname{gl} \operatorname{dim} \Lambda=2$

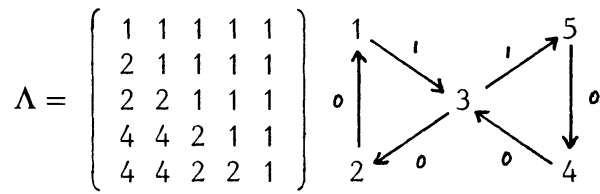

$\operatorname{gl} \operatorname{dim} \Lambda=2$

$d=15$

$d=15$

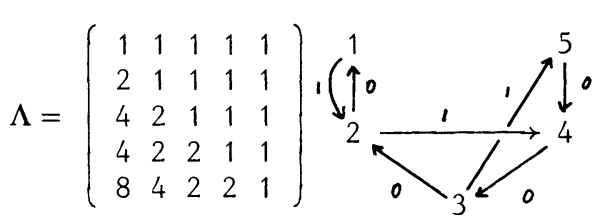

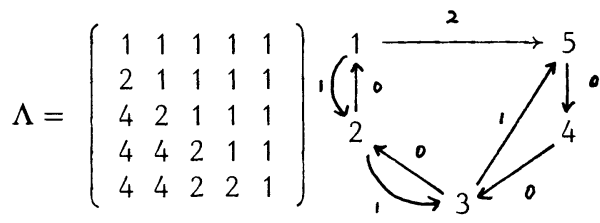

gl.dim $\Lambda=2$

$\operatorname{gl} \operatorname{dim} \Lambda=3$

$d=15$

$d=15$

$\Lambda=\left(\begin{array}{lllll}1 & 1 & 1 & 1 & 1 \\ 2 & 1 & 2 & 1 & 1 \\ 4 & 2 & 1 & 1 & 1 \\ 4 & 2 & 2 & 1 & 1 \\ 4 & 4 & 2 & 2 & 1\end{array}\right),(\underbrace{\stackrel{2}{\longrightarrow}}_{1}$

$\left.\Lambda=\left(\begin{array}{lllll}1 & 1 & 1 & 1 & 1 \\ 2 & 1 & 1 & 1 & 1 \\ 4 & 2 & 1 & 1 & 1 \\ 4 & 2 & 2 & 1 & 1 \\ 4 & 4 & 4 & 2 & 1\end{array}\right),\left(\prod_{0}^{1}, \frac{2}{2}\right\rfloor_{0}^{5}\right)_{0}^{5}$

gl. $\operatorname{dim} \Lambda=4$

gl. $\operatorname{dim} \Lambda=3$

$d=15$

$d=15$

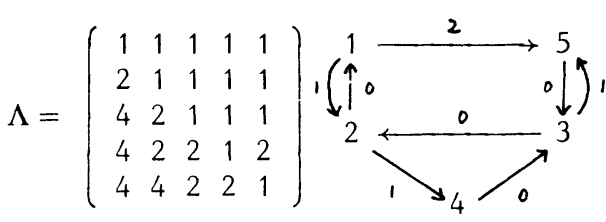

$\Lambda=\left(\begin{array}{lllll}1 & 1 & 1 & 1 & 1 \\ 2 & 1 & 2 & 2 & 1 \\ 4 & 2 & 1 & 1 & 1 \\ 4 & 2 & 2 & 1 & 1 \\ 4 & 2 & 2 & 2 & 1\end{array}\right)$

$\operatorname{gl} \operatorname{dim} \Lambda=3$

$\operatorname{gl} \cdot \operatorname{dim} \Lambda=3$ 
$d=15$

$\Lambda=\left(\begin{array}{lllll}1 & 1 & 1 & 1 & 1 \\ 2 & 1 & 1 & 1 & 1 \\ 4 & 2 & 1 & 2 & 2 \\ 4 & 2 & 2 & 1 & 1 \\ 4 & 2 & 2 & 2 & 1\end{array}\right)$

gl. $\operatorname{dim} \Lambda=2$

$d=16$

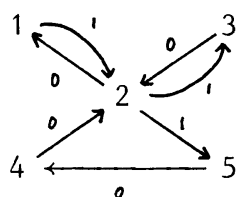

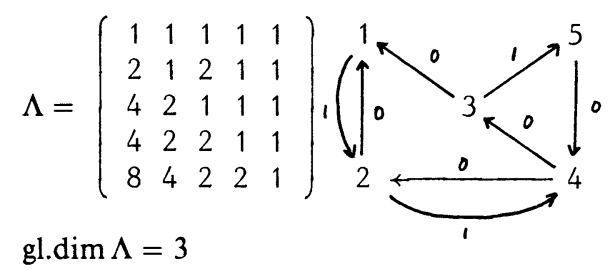

$d=16$

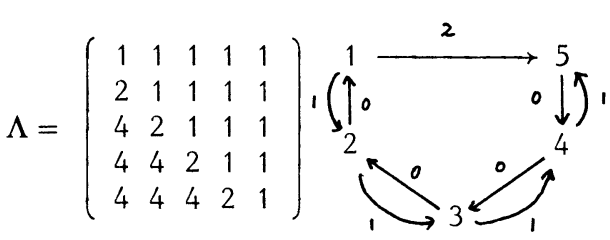

gl.dim $\Lambda=4$

$d=16$

$\Lambda=\left(\begin{array}{lllll}1 & 1 & 1 & 1 & 1 \\ 2 & 1 & 1 & 1 & 1 \\ 4 & 2 & 1 & 1 & 1 \\ 4 & 4 & 2 & 1 & 2 \\ 4 & 4 & 2 & 2 & 1\end{array}\right)=\underset{1}{\stackrel{2}{(}}$

gl.dim $\Lambda=3$

$d=16$

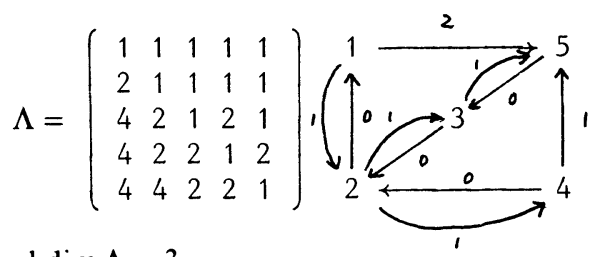

$\operatorname{gl} . \operatorname{dim} \Lambda=3$ $d=16$

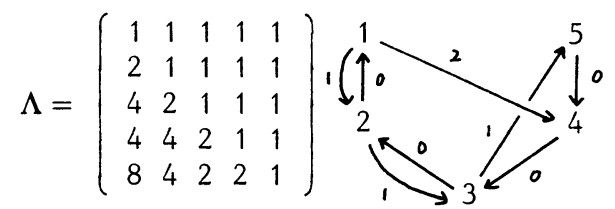

$\operatorname{gl} \operatorname{dim} \Lambda=3$

$d=16$

$\Lambda=\left(\begin{array}{lllll}1 & 1 & 1 & 1 & 1 \\ 2 & 1 & 1 & 1 & 1 \\ 4 & 2 & 1 & 1 & 1 \\ 4 & 2 & 2 & 1 & 1 \\ 8 & 4 & 4 & 2 & 1\end{array}\right),\left(\prod_{0} \underset{0}{\stackrel{2}{2}}\right.$

$\operatorname{gl} \cdot \operatorname{dim} \Lambda=2$

$d=16$

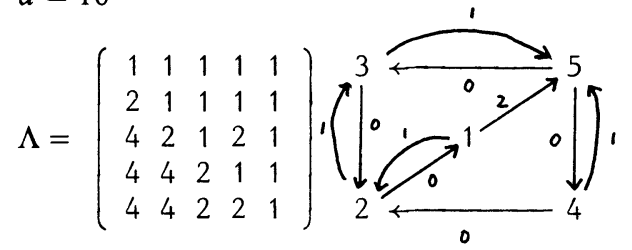

$\operatorname{gl} \cdot \operatorname{dim} \Lambda=3$

$d=16$

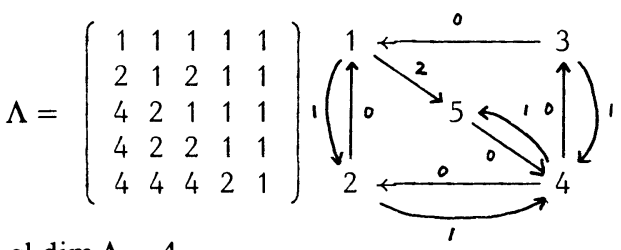

$\operatorname{gl} \operatorname{dim} \Lambda=4$

$d=16$

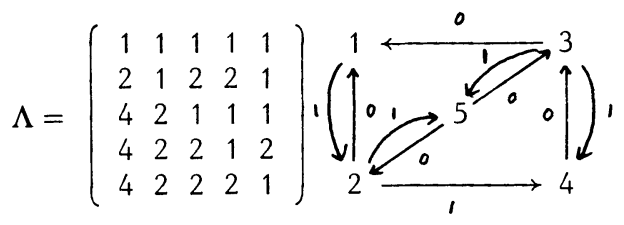

$\operatorname{gl} \cdot \operatorname{dim} \Lambda=3$ 
$d=16$

$d=17$

$\Lambda=\left(\begin{array}{lllll}1 & 1 & 1 & 1 & 1 \\ 2 & 1 & 1 & 1 & 1 \\ 4 & 2 & 1 & 2 & 2 \\ 4 & 2 & 2 & 1 & 2 \\ 4 & 2 & 2 & 2 & 1\end{array}\right)$

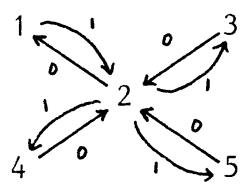

$\operatorname{gl} \operatorname{dim} \Lambda=2$

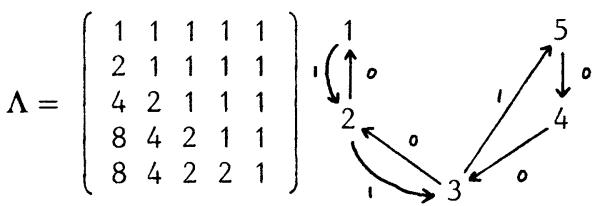

$\operatorname{gl} \operatorname{dim} \Lambda=2$

$d=17$

$d=17$

$\Lambda=\left(\begin{array}{lllll}1 & 1 & 1 & 1 & 1 \\ 2 & 1 & 1 & 1 & 1 \\ 4 & 2 & 1 & 1 & 1 \\ 4 & 4 & 2 & 1 & 1 \\ 8 & 4 & 4 & 2 & 1\end{array}\right)$

gl. $\operatorname{dim} \Lambda=4$

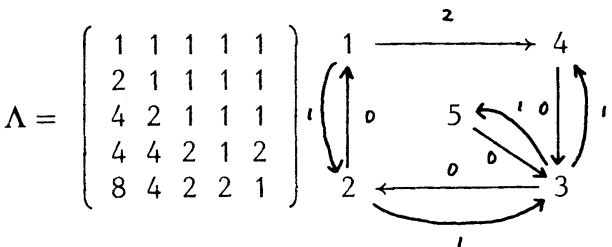

gl.dim $\Lambda=3$

$d=17$

$d=17$

$\Lambda=\left(\begin{array}{lllll}1 & 1 & 1 & 1 & 1 \\ 2 & 1 & 2 & 1 & 1 \\ 4 & 2 & 1 & 1 & 1 \\ 4 & 2 & 2 & 1 & 1 \\ 8 & 4 & 4 & 2 & 1\end{array}\right),\left(\left.\right|_{0} ^{1+0}\right.$

gl.dim $\Lambda=3$

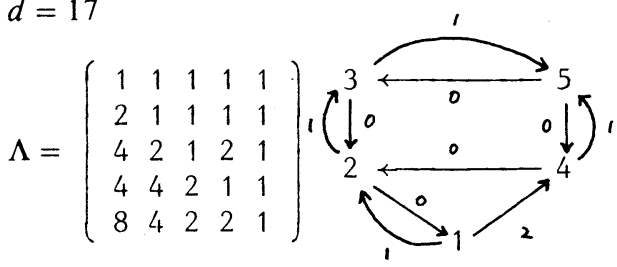

$\operatorname{gl} \cdot \operatorname{dim} \Lambda=4$

$\Lambda=17$

$d=18$

gl.dim $\Lambda=4$

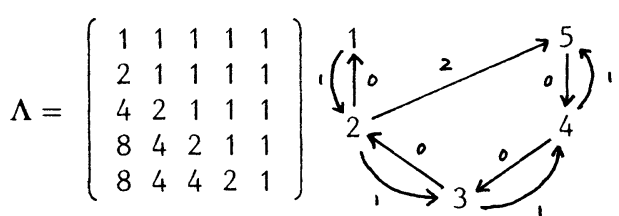

$\operatorname{gl} \operatorname{dim} \Lambda=3$

$d=18$

$d=18$

$\Lambda=\left(\begin{array}{lllll}1 & 1 & 1 & 1 & 1 \\ 2 & 1 & 1 & 1 & 1 \\ 4 & 2 & 1 & 2 & 1 \\ 8 & 4 & 2 & 1 & 1 \\ 8 & 4 & 2 & 2 & 1\end{array}\right)$

$\Lambda=\left(\begin{array}{lllll}1 & 1 & 1 & 1 & 1 \\ 2 & 1 & 1 & 1 & 1 \\ 4 & 2 & 1 & 1 & 1 \\ 8 & 4 & 2 & 1 & 2 \\ 8 & 4 & 2 & 2 & 1\end{array}\right)$

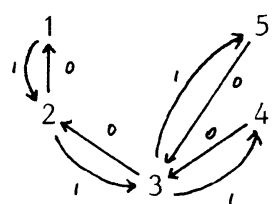

$\operatorname{gl} \cdot \operatorname{dim} \Lambda=3$

gl. $\operatorname{dim} \Lambda=2$ 
$d=19$

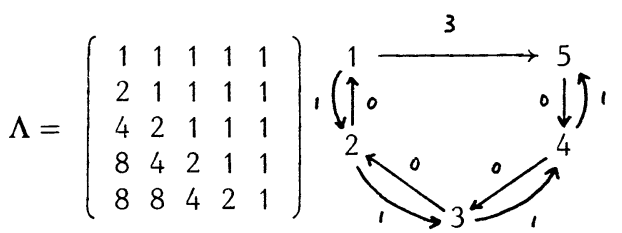

$\operatorname{gl} \operatorname{dim} \Lambda=3$ $d=20$

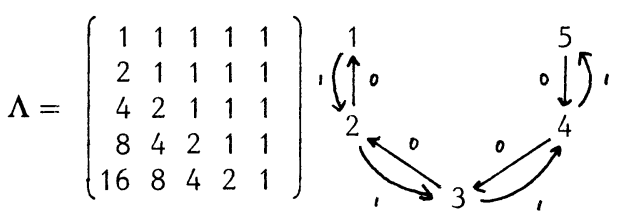

$\operatorname{gl} \operatorname{dim} \Lambda=2$

\section{REFERENCES}

1. H. Bass, Finitistic dimension and a homological generalization of semiprimary rings, Trans. Amer. Math. Soc. 95 (1960), 466-488.

2. H. Fujita, An idealizer with respect to a link between maximal ideals, Comm. Algebra $\mathbf{1 6}$ (1988), 1053-1082.

3. K. R. Goodearl, Subrings of idealizer rings, J. Algebra 33 (1975), 405-429.

4. V. A. Jategaonkar, Global dimension of triangular orders over a discrete valuation ring, Proc. Amer. Math. Soc. 38 (1973), 8-14.

5. __ Global dimension of tiled orders over a discrete valuation ring, Trans. Amer. Math. Soc. 196 (1974), 313-330.

6. E. Kirkman and J. Kuzmanovich, Global dimension of a class of orders, preprint, Wake Forest Univ., 1988.

7. J. C. Robson, Idealizers and hereditary noetherian prime rings, J. Algebra 22 (1972), 45-81.

8. R. Tarsy, Global dimension of orders, Trans. Amer. Math. Soc. 151 (1970), 335-340.

9. A. Wiedemann and K. W. Roggenkamp, Path orders of global dimension two, J. Algebra 80 (1983), 113-133.

InStitute of Mathematics, University of Tsukuba, Ibaraki 305, Japan 